\title{
Reliability Sensitivity Analysis of Gear Reducer Based on Probabilistic Design System
}

\author{
Cao Tong ${ }^{1,2}$, Yuanzheng Tian ${ }^{1,2}$, Changshuai $\mathrm{Yu}^{1,2}, \mathrm{Yu} \mathrm{Xing}^{1,2}$ \\ 1. State Key Laboratory of Robotics, Shenyang Institute of Automation, Chinese Academy of Sciences, Shenyang, China \\ 2. Institutes for Robotics and Intelligent Manufacturing, Chinese Academy of Sciences, Shenyang, China \\ tongcao19@163.com,297410219@qq.com,1085076990@qq.com,2603580518@qq.com
}

\begin{abstract}
In order to quantitatively reflect the influence degree of various random factors on the reliability of the reducer system, the gear reducer is taken as the object of research in this paper. Firstly, the gear contact fatigue is taken as the main failure mode, and then the tooth contact stress response is calculated by ANSYS/LS-DYNA. As a result, the response function is established based on the response surface method (RSM); secondly, Monte Carlo simulation with 2000 samples were carried out through the probabilistic design system (PDS) module of ANSYS, so that the influence of bearing clearance, gear's pitch error and tooth width machining error on tooth surface contact failure was obtained. This study provides an important theoretical basis for the optimal design of the reducer.
\end{abstract}

Keywords-gear wheel reducer; response surface; tooth surface contact; reliability sensitivity; PDS

\section{Introduction}

The reducer is the most important power transmission and motion transmission device. It is widely used in metallurgy, mining, transportation, cement, construction, chemical, textile, printing and dyeing, pharmaceutical and other fields. And it plays a great significance on the national economy.

Studies have shown that [1-4], random factors of manufacturing and assembling have a lot of influence on the machine. If the sensitivity of the structural parameters and random parameters on the system's reliability is known, mechanical designer can appropriately control the precision according to economy. Therefore, the reliability sensitivity analysis of typical mechanical product can provide a theoretical basis for the reliability design and optimization, and provide a guidance for typical structural product design and manufacturing cost.

In order to quantitatively reflect the influence of random factors such as assembling error, manufacturing error and external load on the fatigue reliability of gear transmission. In this paper, the gear transmission reducer is taken as the research object. The reliability analysis of the probabilistic design system (PDS) technology based on ANSYS is carried out to provide a theoretical basis for gear reliability optimization design.

\section{Reliability sensitivity analysis method}

\section{A. Response Surface Method(RSM)}

The idea of response surface method is to replace a real limit state surface by fitting a response surface through a series of deterministic experiments. The response surface can be simulated by a mathematical equation. It is assumed that the relationship between $Z$ and the random parameter vector $\boldsymbol{X}$ $=\left[X_{1}, X_{2}, \ldots, X_{N R}\right]$ can be expressed as the quadratic function, as is shown in the following equation

$$
\hat{Z}=a_{0}+\sum_{i=1}^{N R} a_{i} X_{i}+\sum_{i=1}^{N R} \sum_{j=i}^{N R} a_{i j} X_{i} X_{j}
$$

In formula (1), $a_{0} 、 a_{i}, a_{i j}(i=1 \ldots N R ; j=i \ldots N R)$ is the undetermined coefficient. This paper uses the matrix experimental design method. The method takes three horizontal points for each random variable, and then combines the center point and the side midpoint according to BoxBehnken as sample points [5].

For random variables with arbitrary distribution, the horizontal point value $x_{\mathrm{s}}$ of random variables can be determined by equation (2).

$$
\int_{-\infty}^{x_{s}} f(x) \mathrm{d} x=p_{n}, \quad n=1,2,3
$$

where $f(x)$ is the distribution density function of the random variable; $p_{n}$ is the level, taking $p_{1}=0.01 、 p_{2}=0.5 、 p_{3}=0.99$.

For the random variable with the normal distribution, then

$$
x_{\mathrm{s}}=\mu+\sigma \Phi^{-1}\left(p_{n}\right)
$$

Regression analysis of $N S$ output points $\left(z_{1}, z_{2}, \ldots, z_{N S}\right)$ by least squares method, then

$$
\begin{aligned}
s=\sum_{i=1}^{N S} \varepsilon^{2}=\sum_{i=1}^{N S}\left[z_{i}-\left(a_{0}+\sum_{i=1}^{N R} a_{i} x_{i}+\sum_{i=1}^{N R} \sum_{j=i}^{N R} a_{i j} x_{i} x_{j}\right)\right]^{2} \\
\qquad \begin{array}{l}
\frac{\partial s}{\partial a_{0}}=0 \\
\frac{\partial s}{\partial a_{i}}=0, i=1,2, \cdots, N R \\
\frac{\partial s}{\partial a_{i j}}=0, i=1,2, \cdots, N R ; j=i, \cdots, N R
\end{array}
\end{aligned}
$$


The values of the coefficients in the equation (1) can be obtained, thereby the response surface function is constructed. The response surface function can then be used to replace the real response for reliability analysis.

\section{B. Sensitivity Analysis Based on RSM-MonteCarlo and PDS}

In order to obtain the influence of random variables on the limit state equation of the reducer, the sensitivity of the limit state function to the structural parameters (such as size, diameter, width, etc.) is characterized and explained, thus providing guidance and reference value for improving the structure.

Through the reliability sensitivity analysis module of ANSYS probabilistic design system (PDS) technology, the analysis code of component response surface function is written by APDL, and the influence of various factors on the limit state equation is effectively analyzed. In the analysis process, each random parameter is described as an uncertainty variable obeying a certain probability density distribution, and the distribution characteristics, influence relationship and influence degree of each response parameter are analyzed through a large number of sampling points. Parameter sensitivity. The PDS probability design process is shown in Fig. 1. Main steps: Select experimental design, virtual simulation test, establish response surface function, select analysis tool, specify reliability analysis file, cycle sampling, and post-process result.

\section{Gear reducer reliability sensitivity analysis}

\section{A. Determination of Random Factors}

Assembling error and manufacturing error are the main factors of contact fatigue reliability analysis, mainly came from the non-parallelism of the support shaft of the housing, the center-to-center deviation of the two shafts, the radial clearance of the bearing, the installation error and other factors. In the research of this subject, the radial clearance of the cylindrical roller bearing in the shafting system of the shaft gear is used as the structural improvement parameter. Since the cylindrical roller bearing clearance group is grade 1, the bearing design manual is obtained to obtain the minimum and maximum limits, which are $10 \mu \mathrm{m}$ and $20 \mu \mathrm{m}$, respectively.

Then according to the $3 \sigma$ rule, bring $\Phi^{-1}\left(p_{1}\right)=-3$, $\Phi^{-1}\left(p_{2}\right)=0, \Phi^{-1}\left(p_{3}\right)=3$ into equation (2) and formula (3), then the mean $\mu$ and standard deviation $\sigma$ data is obtain as follow

$$
\left\{\begin{array}{l}
\mu=\left(x_{3}-x_{1}\right) / 2 \\
\sigma=\left(x_{3}-x_{1}\right) / 6
\end{array}\right.
$$

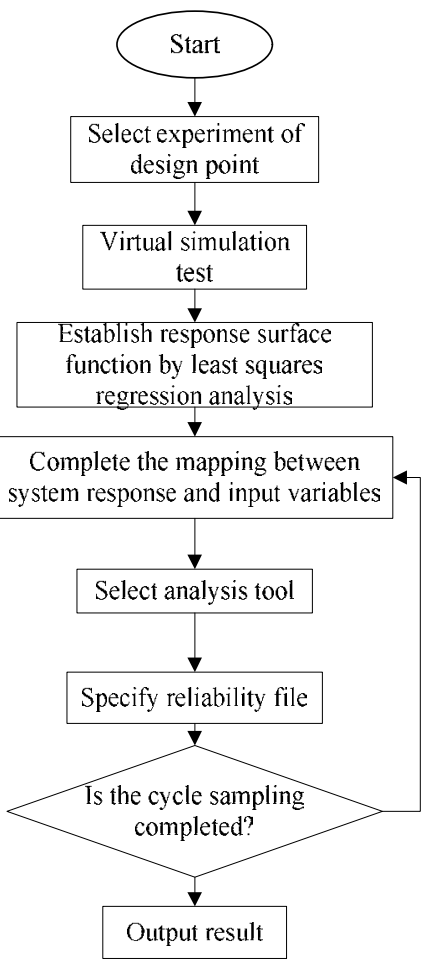

Fig. 1. Calculation flow chart of PDS.

The reduction's gear machining accuracy is grade 6 , the minimum pitch deviation and the maximum limit value are -9 $\mu \mathrm{m}$ and $9 \mu \mathrm{m}$, respectively, and the tooth width limit deviation is $-7.5 \mu \mathrm{m}$ and $7.5 \mu \mathrm{m}$. According to the $3 \sigma$ rule, the mean $\mu$ and standard deviation $\sigma$ data are calculated by the equation (10), as shown in Table 1.

TABLE I. MEAN AND STANDARD DEVIATION OF EACH PARAMETER

\begin{tabular}{|c|c|c|c|}
\hline Parameter & $\begin{array}{c}\text { Distribution } \\
\text { type }\end{array}$ & Mean & $\begin{array}{l}\text { Standard } \\
\text { deviation }\end{array}$ \\
\hline $\begin{array}{l}\text { Pitch error } \\
\Delta_{\text {Pitch }} / \mu \mathrm{m}\end{array}$ & Gauss & 0 & 3 \\
\hline $\begin{array}{c}\text { Radial } \\
\text { clearance } Y / \mu \mathrm{m}\end{array}$ & Gauss & 15 & 3.3 \\
\hline $\begin{array}{c}\text { Tooth width } \\
B / \mu \mathrm{m}\end{array}$ & Gauss & 20 & 2.5 \\
\hline
\end{tabular}

\section{B. Establishment of System's Response Surface Function}

The finite element calculation of the pinion was carried out by ANSYS/LS-DYNA software. The result is shown in Fig. 2. The maximum contact stress of the gear tooth surface is 226.743 $\mathrm{MPa}$. The system response values corresponding to the sample points are calculated according to the BoxBehnken sampling method and the virtual simulation are listed in Table 2.

It is assumed that the maximum contact stress of the gear follows a normal distribution and the parameters are estimated. The gear material is $12 \mathrm{CrNi}$. When the life of the high-speed shaft is $1 \times 10^{6}$, the allowable contact strenth $\left[\sigma_{H}\right]$ of the pinion is $2089.2 \mathrm{MPa}$, and the response value is obtained after 13 times of finite element virtual numerical simulation according 
to the sample point. Enter the last column of Table 2 . According to formula (1), the response surface function of gear contact stress is obtained as

$$
\begin{aligned}
& g=407.825+0.094444 \Delta_{\text {Pitch }}-2.62500 Y+0.59333 B \\
& +0.047222 \Delta_{\text {Pitch }} Y+0.00037037 \Delta_{\text {Pitch }} B+0.002 Y B \\
& -0.00509259 \Delta_{\text {Pitch }}^{2}+0.10750 Y^{2}-0.007333 B^{2}
\end{aligned}
$$

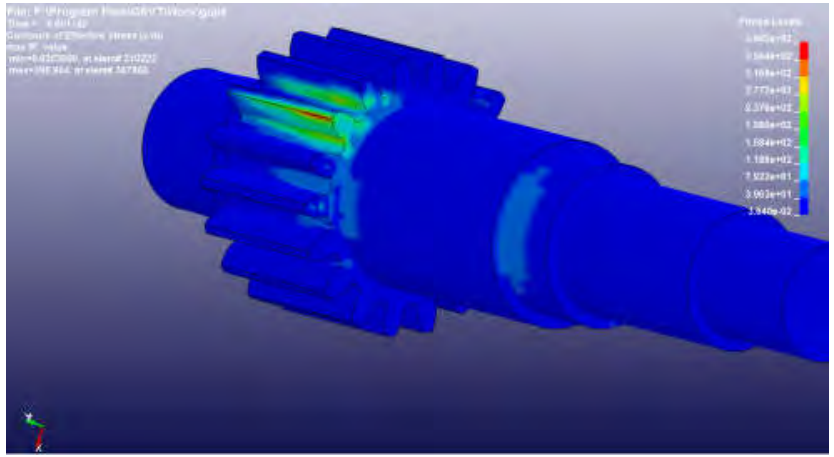

\begin{tabular}{|c|c|c|c|c|c|c|c|c|}
\hline \multirow{2}{*}{ Sample point } & \multicolumn{2}{|c|}{$\Delta_{\text {Pitch }}$} & \multicolumn{2}{|c|}{$Y$} & \multicolumn{2}{|c|}{$B$} & \multirow{2}{*}{ Type } & \multirow{2}{*}{ Max Stress } \\
\hline & Level & Value & Level & Value & Level & Value & & \\
\hline 1 & $p_{2}$ & 0 & $p_{2}$ & 15 & $p_{2}$ & 7.5 & Central point & 396.9 \\
\hline 2 & $p_{1}$ & -9.00 & $p_{1}$ & 10.00 & $p_{2}$ & 7.50 & \multirow{12}{*}{ Mid point } & 388.5 \\
\hline 3 & $p_{3}$ & 9.00 & $p_{1}$ & 10.00 & $p_{2}$ & 7.50 & & 401.4 \\
\hline 4 & $p_{1}$ & -9.00 & $p_{3}$ & 20.00 & $p_{2}$ & 7.50 & & 392.7 \\
\hline 5 & $p_{3}$ & 9.00 & $p_{3}$ & 20.00 & $p_{2}$ & 7.50 & & 414.1 \\
\hline 6 & $p_{1}$ & -9.00 & $p_{2}$ & 15.00 & $p_{1}$ & 0.00 & & 385.2 \\
\hline 7 & $p_{3}$ & 9.00 & $p_{2}$ & 15.00 & $p_{1}$ & 0.00 & & 397.0 \\
\hline 8 & $p_{1}$ & -9.00 & $p_{2}$ & 15.00 & $p_{3}$ & 15.00 & & 395.1 \\
\hline 9 & $p_{3}$ & 9.00 & $p_{2}$ & 15.00 & $p_{3}$ & 15.00 & & 407.0 \\
\hline 10 & $p_{2}$ & 0.00 & $p_{1}$ & 10.00 & $p_{2}$ & 0.00 & & 394.6 \\
\hline 11 & $p_{2}$ & 0.00 & $p_{3}$ & 20.00 & $p_{2}$ & 0.00 & & 398.3 \\
\hline 12 & $p_{2}$ & 0.00 & $p_{1}$ & 10.00 & $p_{3}$ & 15.00 & & 399.9 \\
\hline 13 & $p_{2}$ & 0.00 & $p_{3}$ & 20.00 & $p_{3}$ & 15.00 & & 403.9 \\
\hline
\end{tabular}

Fig. 2. Gear dynamic contact stress.

TABLE II. BOX-BEHNKEN SAMPLE POINT AND RESPONSE VALUE

\section{System reliability sensitivity analysis}

The reliability analysis file is carried out, and then the coefficients between the input variables and response is calculated according to equation (7). The analysis tool and method is selected, so the maximum contact stress of the gear tooth surface is specified as the random output variable. The distribution type, distribution function and each input variable is determined. Therefore, the probability density function and distribution function curve of the output variable are shown in Fig. 3.
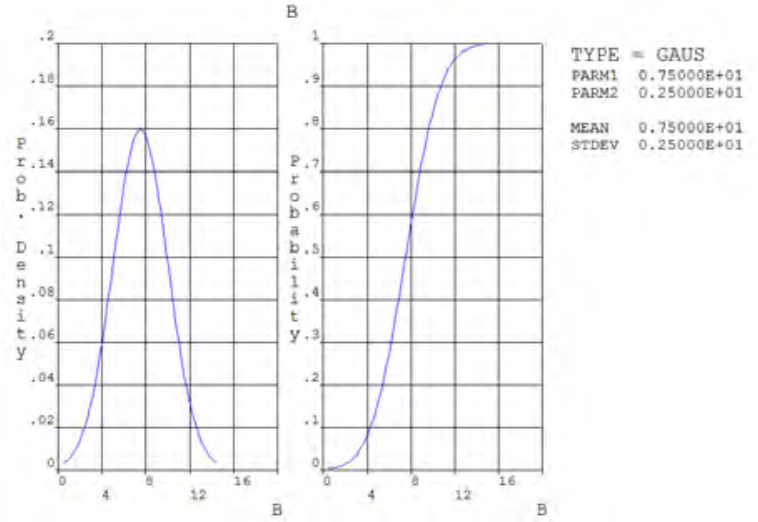

Fig. 3. limit state variable g probability density function and distribution function curve
According to equation (7), the limit state function $g$ is established. And if $g<0$, it is invalid. In the probabilistic design process, the pinion analysis module is sampled 2000 times, and the reliability and sensitivity value of each random parameter are obtained. Fig. 4 is an output variable sample history curve that reproduces the value of 2000 samples.

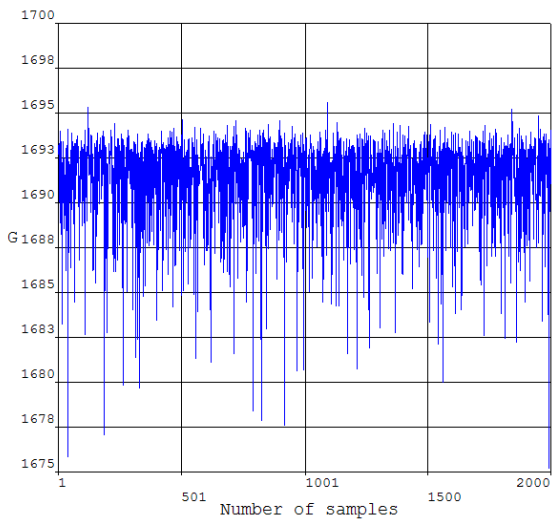

Fig. 4. Limit state variable $\mathrm{g}$ history curve

The histogram is an important result of PDS analysis. The histogram of gear pitch error, bearing radial clearance and gear width is shown in Fig. 5. The histogram is a way to check if the number of loops is sufficient. As can be seen from Fig. 5, the probability function curve close to the normal distribution 
is smooth and has no large gap. Therefore, it indicates that the number of samples is sufficient.
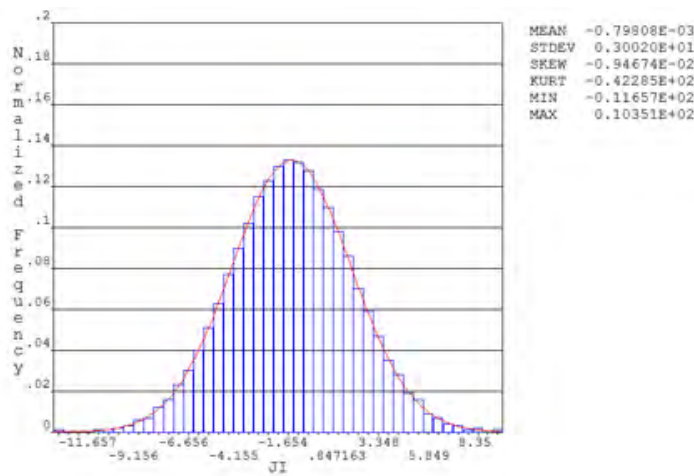

(a) Histogram of gear pitch error $\Delta_{\text {Pitch }}$
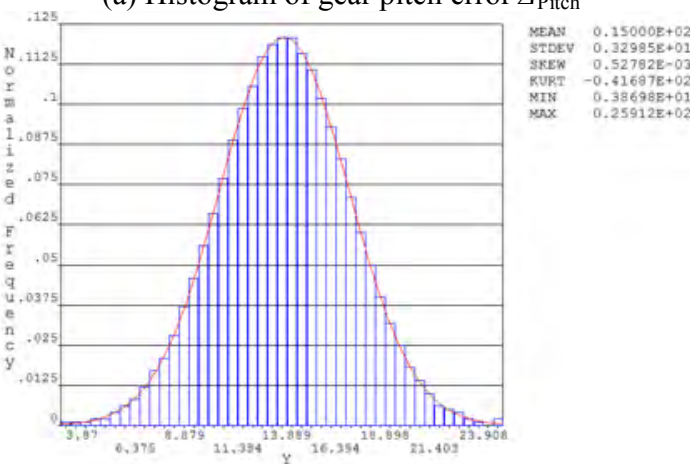

(b) Histogram of bearing radial clearance $Y$

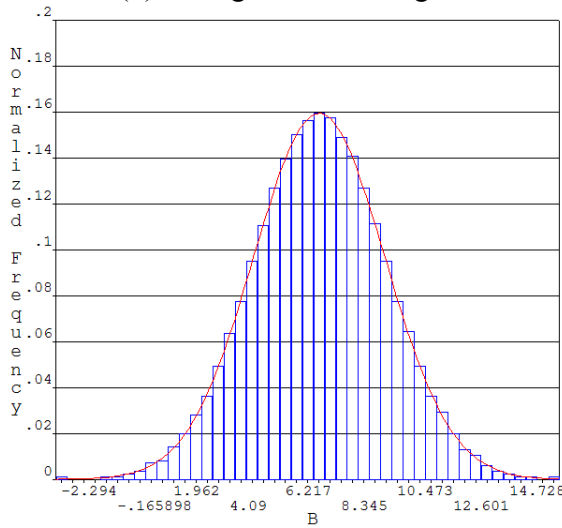

(c) Histogram of gear width $B$

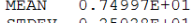
STDEV $\quad 0.25028 \mathrm{E}+01$ KURT $-0.424565+02$ MIN $-0.22937 \mathrm{E}+01$ MAX $\quad 0.16431 \mathrm{E}+02$

Fig. 5. Histogram of the design variable of the reducer

Fig. 6 is a sample history curve of the random output variable $g$, where (a) is the sample mean history of $g$ and (b) is the sample variance history of $g$. The width of the convergence band in the graph indicates whether the number of samples is sufficient. It can be seen that the convergence of the sample mean and the sample variance is relatively narrow, also indicating that the number of samples is sufficient.

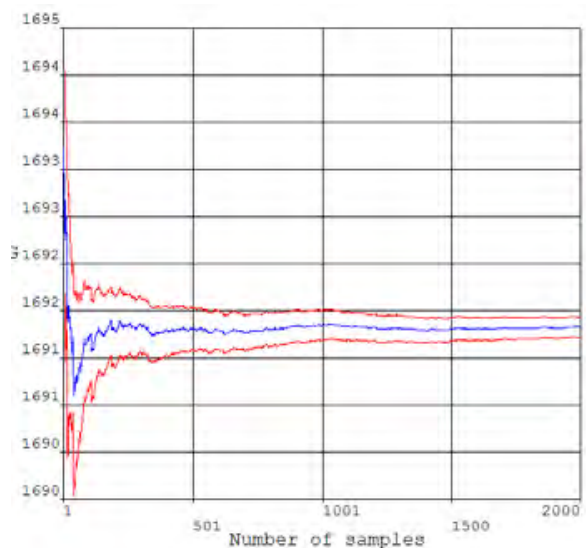

MEAN $\quad 0.16913 \mathrm{E}+04$ STDEV $0.245 \mathrm{~B} 3 \mathrm{E}+01$ $\begin{array}{ll}\text { SKEW } & -0.19886 \mathrm{E}+01 \\ \text { KURT } & -0.11567 \mathrm{E}+03\end{array}$ MIN $\quad 0.16752 \mathrm{E}+04$ Confidence Limit 95.005

(a) Sample mean history of random output variable $g$

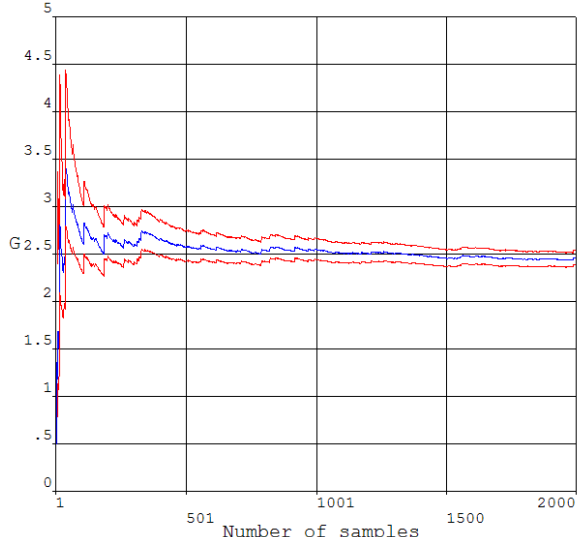

MEAN $\quad 0.16913 \mathrm{E}+04$ STDEV $0.24583 \mathrm{E}+01$ SREW $-0.19886 \mathrm{E}+01$ MTN $0.16752 \mathrm{E}+04$ MIN $\quad 0.16752 \mathrm{E}+04$ Confidence Limit 95.008

(b) Sample standard deviation history of random output variable g Fig. 6. Sample history of the output variable $g$

Fig. 7 is a cumulative distribution function curve for $g$, which reflects the failure probability of gear contact. The value at any point is equal to the probability that the data appears below that point. From the cumulative distribution function curve of $g$, the failure probability of the gear contact with different tooth surface contact strengths can be obtained.
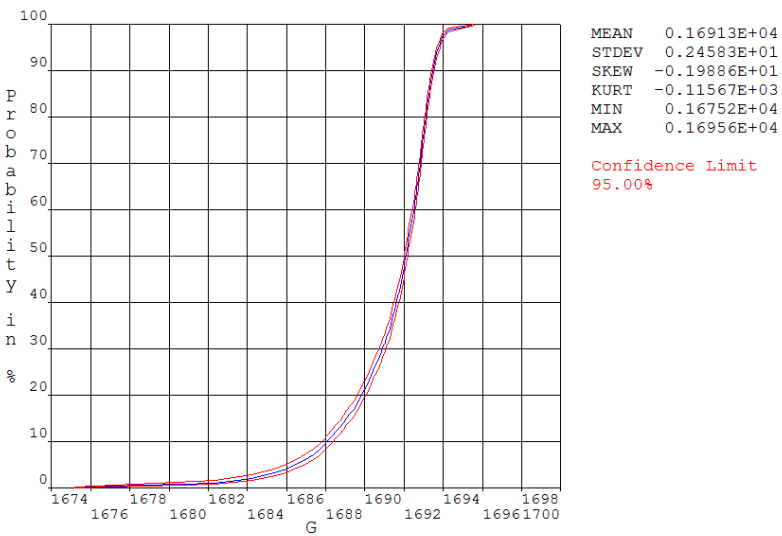

Fig. 7. Cumulative distribution function curve of failure function $g$

Fig. 8 is a calculated reliability diagram of the gear meshing drive reliability. The graph reflects the degree of influence of each random parameter on its reliability with a quantitative probability. The sensitivity map indicates the 
reliability sensitivity of each parameter in a bar graph or a pie chart. The input variable with the highest sensitivity in the bar graph appears at left, and the others are arranged to the right. Positive and negative signs indicate whether the input and output variables change in the same trend. The most important input variable in the pie chart appears at 12 o'clock after the clock surface, and the others are sequentially arranged in a clockwise direction. The sensitivity values of each parameter are shown in Fig. 8. The maximum sensitivity is the bearing clearance, which is -0.721 . the deviation of the gear pitch error is -0.346 , and the tooth width is 0.273 . The deviation between the clearance and the pitch is negative, indicating that the output variable and reliability decreases when their value increase; The tooth width sensitivity is positive, indicating that the parameter increases with the value increasing.

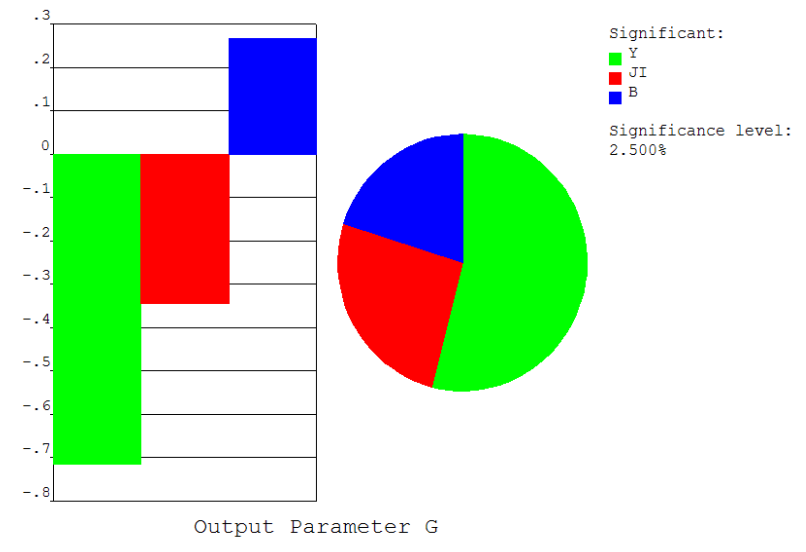

Fig. 8. Sensitivity of each random parameter

From the pie chart of the reliability sensitivity of each parameter obtained from PDS, it can be seen that the reduction's the tooth width error will make the reliability to be more unreliable (failure), but the tooth width error to the failure of the gear contact failure isn't less sensitive. So the tooth width can be appropriately reduced, the speed reducer system can be made lighter, and the machining accuracy of width can be appropriately relaxed to reduce the manufacturing cost.

The above numerical calculation results can provide a basis for the engineering designer to accurately design the gear reducer. The above calculation results are completely consistent with the usual qualitative analysis', which further demonstrates the comprehensiveness and correctness of the sensitivity matrix to the analysis of various factors of the reducer gear.

\section{Conclusions}

(1) The tooth contact stress response function is established by the response surface method, thereby the problem of implicit function and large calculation is solved.

(2) The ANSYS probability analysis module PDS can deal the probability analysis problem very well. By simulating the Monte Carlo values with 2000 samples, result shows that the response variable histogram is smooth and small, and the convergence of sample mean and sample variance is narrow. The number of samples is sufficient.

\section{Acknowledgment}

This research was financially supported by the State Key Laboratory of Robotics (Grant No. 2017-Z18) and Liaoning Provincial Natural Science Foundation (Grant No. 2018010334-301). Their financial supports are gratefully acknowledged.

\section{References}

[1] L. Runfang, W. Jianjun. Gear system dynamics. Shanghai: Science Press, pp. 59-68, 1997.

[2] K. J. Huang, C. C. Liang, J. Y. Chen. Time varying approaches to dynamic analysis of a planetary gear system using a discrete and a continuous models. Detc2007:Proceedings of the Asme International Design Engineering Technical Conferences and Computers and Information in Engineering Conference, vol. 7, issue 2, pp. 451-458, 2008.

[3] M. Ristivojevic, Lazovic T, Vencl A, Studying the load carrying capacity of spur gear tooth flanks. Mechanism and Machine Theory, vol. 59, issue 1, pp. 125-137, 2013.

[4] T. Cao, S. Zhili, M. Xiaoying, C. Xiaodong. Dynamic simulation of spur gears with assembly errors and machining errors. Dongbei Daxue Xuebao/Journal of Northeastern University, vol. 35, issue 7, pp. 996$1000,2014$.

[5] Amstadter BL. Reliability Mathematics[M], New York: John Wiley \& Sons, 1977.

[6] S. Zhili, C. Liangyu. Practical mechanical reliability design theory and method, Beijing: Science Press, pp. 210-211, 2003.

[7] S. T. Li. Effects of machining errors, assembly errors and tooth modifications on loading capacity, load-sharing ratio and transmission error of a pair of spur gears. Mechanism and Machine Theory, vol. 42, issue 6, pp. 698-726, 2007.

[8] S. Rahman. Probabilistic Elastic-Plastic Fracture Analysis of Circumferentially Cracked Pipes with Finite-Length Surface Flaws. Nuclear Engineering and Design, vol. 195, pp. 698-726, 2007. 